\title{
LncRNA MIR31HG is induced by tocilizumab and ameliorates rheumatoid arthritis fibroblast-like synoviocyte-mediated inflammation via miR-214-PTEN-AKT signaling pathway
}

\author{
Liang $\mathrm{Cao}^{1,2,{ }^{*}, \text { Haifeng Jiang }}{ }^{3,}$, Jing Yang ${ }^{1,}{ }^{*}$, Jun $\mathrm{Mao}^{2}$, Guofeng $\mathrm{Wei}^{4}$, Xiangyun Meng ${ }^{2}$, \\ Hongmei Zang ${ }^{1}$
}

${ }^{1}$ School of Pharmacy, Anhui Medical University, Hefei, Anhui, China

${ }^{2}$ Department of Pharmacy, The Second People's Hospital of Hefei, Hefei, Anhui, China

${ }^{3}$ Institute of Clinical Pharmacology, Anhui Medical University, Hefei, Anhui, China

${ }^{4}$ Department of Emergency, The Second People's Hospital of Hefei, Hefei, Anhui, China

*Equal contribution

Correspondence to: Xiangyun Meng, Hongmei Zang; email: mengxiangyun@ahmu.edu.cn, zanghongmei@ahmu.edu.cn Keywords: RA-FLS, tocilizumab, MIR31HG, miR-214, PTEN

Received: May 18, $2021 \quad$ Accepted: September 7, $2021 \quad$ Published: November 9, 2021

Copyright: (C) 2021 Cao et al. This is an open access article distributed under the terms of the Creative Commons Attribution License (CC BY 3.0), which permits unrestricted use, distribution, and reproduction in any medium, provided the original author and source are credited.

\section{ABSTRACT}

Fibroblast-like synoviocytes (FLS) obtained from the joint synovium of rheumatoid arthritis (RA) patients exhibit hyperplasia and aggressive inflammatory phenotypes. This study was designed to explore the antiinflammatory mechanism of IL-6R inhibitor, tocilizumab, in FLS-mediated inflammation in RA from the perspective of non-coding RNAs (ncRNAs). To this end, we sorted primary FLS obtained from the synovium of patients with RA and cultured them in vitro. The cells were then treated with tocilizumab and subjected to IncRNA- and miRNA-seq to identify the ncRNAs regulated by tocilizumab treatment using bioinformatic analysis and experimental verification. Tocilizumab treatment enhanced the expression of IncRNA MIR31HG and reduced that of micoRNA-214 (miR-214). In addition, miR-214 activated the AKT signaling pathway by directly targeting MIR31HG and PTEN. In addition, the tocilizumab-MIR31HG-miR-214-PTEN-AKT axis regulated the proliferation, migration, and production of inflammatory molecules and matrix metalloproteinases (MMPs) in RA-FLS. Furthermore, co-culture experiments showed that this axis could inhibit the inflammatory phenotype of macrophages and protect chondrocytes. In summary, our study shows that tocilizumab suppresses RA-FLS inflammation by regulating the MIR31HG-miR-214-PTEN-AKT pathway, and presents new insights on RA pathogenesis and potential targets for RA therapy.

\section{INTRODUCTION}

Rheumatoid arthritis (RA) is a chronic autoimmune disease characterized by synovitis, autoantibody production, and erosion of cartilage that severely affect the joints [1]. Although significant progress has been achieved in developing effective RA therapies, challenges of resistance to certain RA drugs and their toxicity remain [2]. Thus, elucidating the pathogenesis of RA and the development of effective therapies remains a priority.

The joint synovium includes the lining and subliming layers, which contain a variety of cells including the fibroblast-like synoviocytes (FLS) [3], synovial macrophages [4], T cells [5] and B cells [6], with the FLS being the most abundant cell type. Normal synovial tissue is characterized by a thin lining 
consisting of several layers of cells. However, in $\mathrm{RA}$, this lining becomes inflamed, hyperplasic, and invades both the cartilage and bone. RA-FLS exhibit aggressive tumor-like phenotypes that can further exacerbate joint damage by invading the extracellular matrix (ECM) [7]. The mechanisms underlying this hyperplasia and cartilage erosion are not fully understood, and thus, further research studies are warranted.

Tocilizumab is approved for use on its own or in combination with other medications to treat moderate to severe RA in adults in whom at least one other medication has failed [8]. However, the specific effects of tocilizumab on RA-FLS inflammation and the related mechanisms remain unknown. Several recent studies have demonstrated the essential role of non-coding RNAs (ncRNAs) in the establishment and development of RA [9]. Thus, this study was designed to explore this area further. We found that tocilizumab inhibited FLS-mediated inflammatory phenotypes by inducing MIR31HG expression in the RA-FLS. Moreover, MIR31HG inhibited the proliferation, migration, and expression of inflammatory cytokines and MMPs by modulating the downstream miR-214-PTEN-AKT pathway in these cells. These findings possibly explain the mechanism underlying the action of this therapy in RA-FLS and proposes novel therapeutic targets for RA.

\section{RESULTS}

\section{Tocilizumab mitigates FLS-mediated inflammation in RA}

We first isolated primary FLS from RA tissues and then cultured them in vitro to assess the effects of tocilizumab treatment on RA-FLS associated inflammation (Figure 1A). Tocilizumab inhibited the proliferation and migration of RA FLS (Figure 1B, 1C) and significantly inhibited the expression of various inflammatory cytokines and matrix metalloproteinases (MMPs) in these cells (Figure 1D, 1E). Our results suggest that tocilizumab treatment may reduce FLSmediated inflammation in RA. Next, we aimed to identify the IncRNAs and miRNAs regulated by tocilizumab in these cells by performing lncRNA- and miRNA-seq (Figure 1F, 1G). After reviewing the data for each of the tocilizumab-induced IncRNA and tocilizumab-inhibited miRNA using bioinformatic analysis and available scientific literature, several lncRNAs (MIR31HG, SNHG5, PCA3) and miRNAs (miR-214, miR-361, miR-575) were selected for experimental validation (Figure $1 \mathrm{H}$ and Supplementary Figures 1, 2).

\section{MIR31HG, a IncRNA targeted by tocilizumab, suppresses RA-FLS inflammation}

Combining analysis of RNA-seq data, qPCR validation (Supplementary Figure 1A) and literature screening, the effects of MIR31HG, SNGH5 and PCA3 on RA-FLS proliferation were evaluated (Supplementary Figure 2) by CCK- 8 assay. The results showed that only MIR31HG KD repress RA-FLS proliferation among these candidates. This analysis identified MIR31HG as a key tocilizumab-induced lncRNA and miR-214 as the key miRNA suppressed by tocilizumab in RA-FLS (Supplementary Figure 1B). Since MIR31HG is a validated target of miR-214 [10], we speculated that tocilizumab may regulate the MIR31HG-miR-214 axis in RA-FLS. Our qPCR results verified that tocilizumab treatment significantly increased the expression of MIR31HG in RA-FLS (Figure 2A). Knockdown of endogenous MIR31HG expression using specific siRNA (Supplementary Figure 3) increased the proliferation, metastasis, and production of inflammatory molecules and MMPs in RA-FLS (Figure 2B-2E). Furthermore, co-culture experiments showed that MIR31HG knockdown in RA-FLS indirectly promoted acquisition of inflammatory phenotypes by the macrophages and aggravated chondrocyte damage (Figure 2F-2J). Taken together, these data suggest that MIR31HG inhibits RA-FLS-mediated inflammation.

\section{The MIR31HG-miR-214-PTEN axis inhibits RA- FLS inflammation}

RNA-seq was used to study the function of miR-214 in RA-FLS. KEGG pathway analysis revealed that miR214 overexpression closely associated with increase in the expression of several genes associated with multiple inflammatory signaling pathways in RA-FLS (Supplementary Figure 4). In addition, miR-214 expression was upregulated in RA synovium compared to the OA control (Figure 3A). Endogenous miR-214 expression was significantly downregulated in RA-FLS treated with tocilizumab (Figure 3B). In addition, siRNA mediated knockdown of MIR31HG led to the upregulation of miR-214 expression in RA-FLS (Figure $3 \mathrm{C}$ ). The results of the bioinformatic analysis, qPCR, western blotting, and luciferase reporter assays demonstrated that miR-214 directly targeted MIR31HG (Figure 3D-3F), as well as PTEN, another antiinflammatory target (Figure 3G-3I). In addition, negative correlations were observed between the expression of miR-214 and MIR31HG/PTEN in these cells (Supplementary Figure 5). Western blot analysis also confirmed that endogenous expression of PTEN was also inhibited in response to MIR31HG knockdown in RA-FLS (Figure 3J), suggesting that MIR31HGmiR-214-PTEN forms a regulatory network in RA-FLS. 

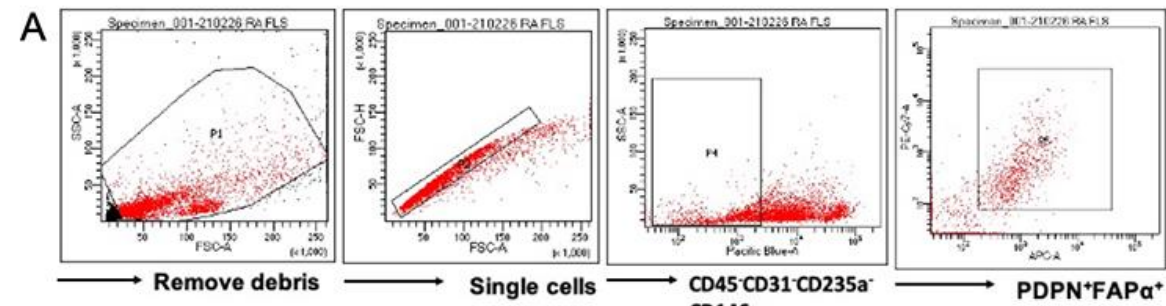

CD146.

B

Proliferation

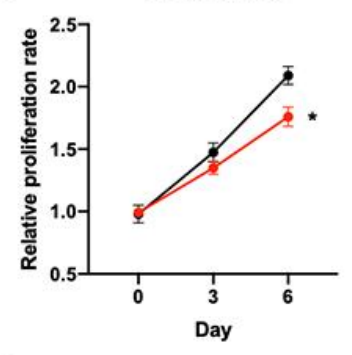

$\rightarrow$ Vehicle control

$\rightarrow$ Tocilizumab
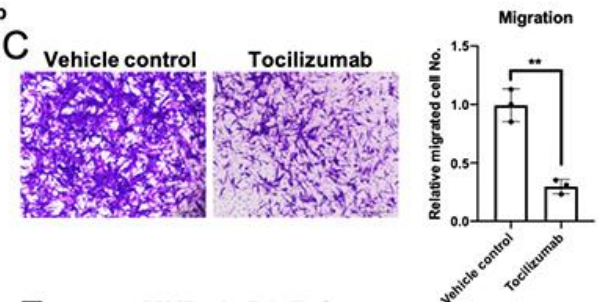

D

RA-FLS

E MMPs in RA-FLS

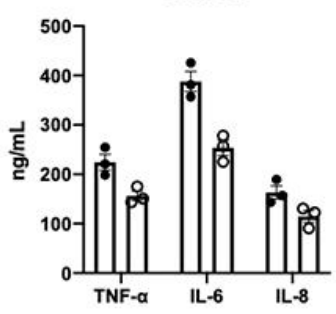

- Control

- Tocilizumab
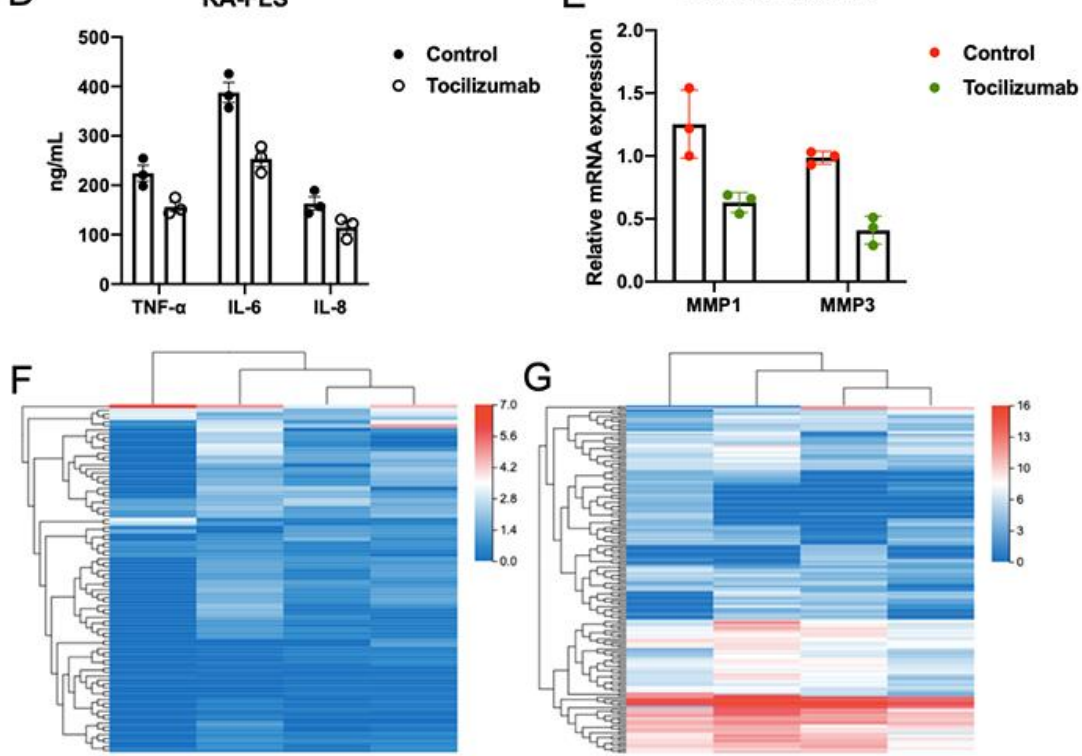

H Tocilizumab-induced IncRNAs in FLS from RA patients

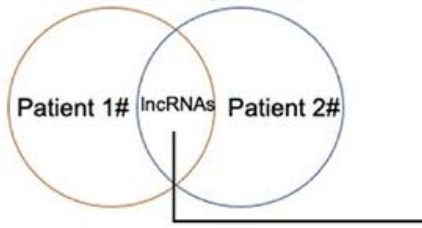

Tocilizumab-repressed miRNAs in FLS from RA patients

Tocilizumab

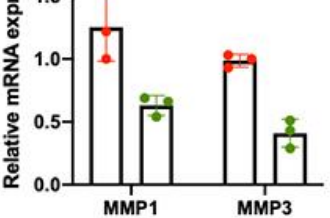


In addition, overexpression of miR-214 promoted the proliferation, migration, and expression of inflammatory molecules and MMPs in primary FLS obtained from patients with RA (Figure 4A-4D). Similar to the MIR31HG knockdown, co-culture experiments showed that miR-214 overexpression indirectly promoted inflammatory phenotypes in the macrophages and increased chondrocyte damage (Figure 4E-4I).

\section{The tocilizumab-MIR31HG axis suppresses the AKT pathway in RA-FLS}

Based on the results from previous reports [11-13] and our RNA-seq results (Supplementary Figure 4), we identified the PI3K/AKT pathway as a potential downstream target of the tocilizumab-MIR31HG-miR214-PTEN axis in RA-FLS. Western blot results
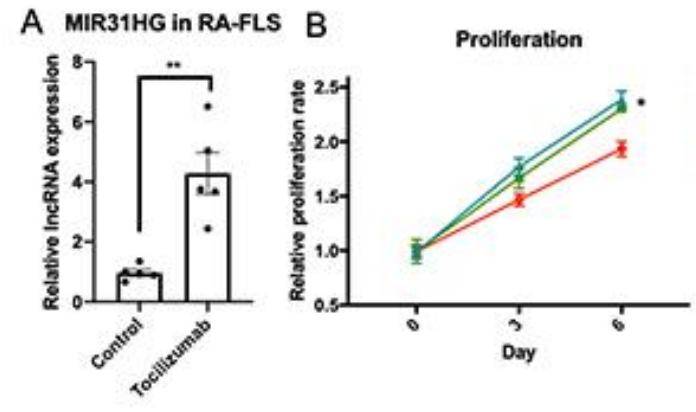

- SiRNANC

- MIR3IHG SIRNA 1A

- MiR3iHg SIRNA 2"
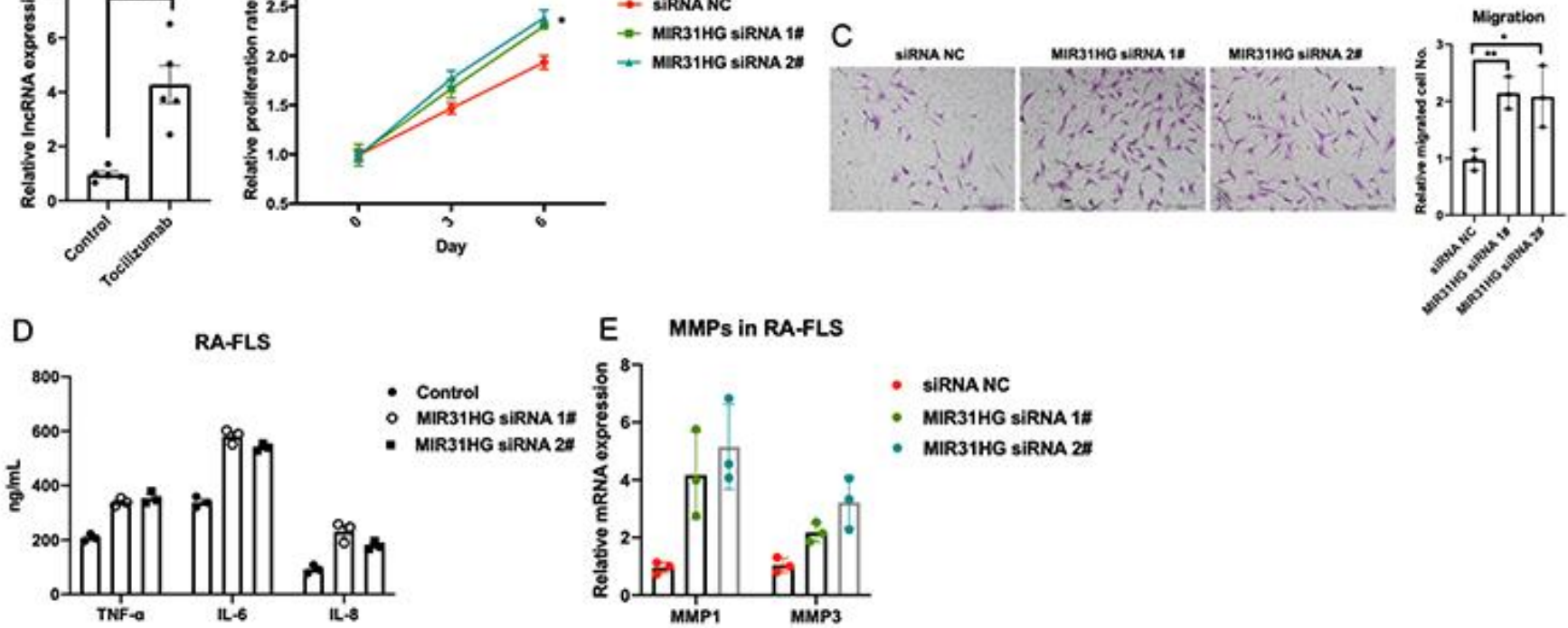

E MMPs in RA-FLS

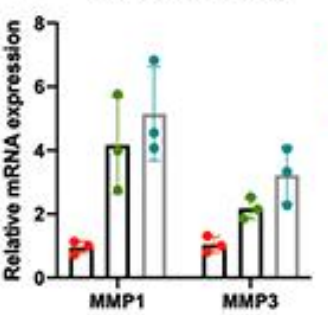

- siRnanc

- mir31hg sirna 1\#

- Mir3ihg siRna zI

MiR31hg siRna 1

- MiR31HG siRea 2

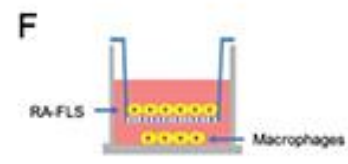

G
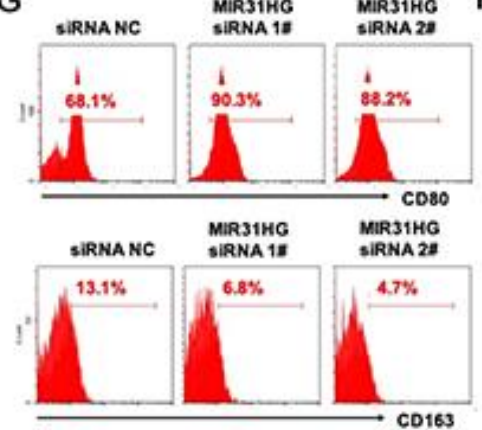

H Polarization of synovial macrophages

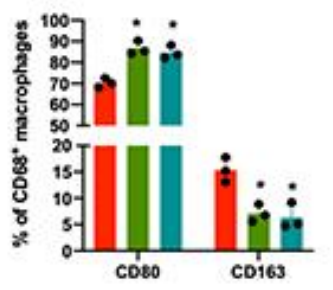

- sirnanc

- MiR31HG SiRNA 1"

- Mir31hg sirnaza

Chondrocytes
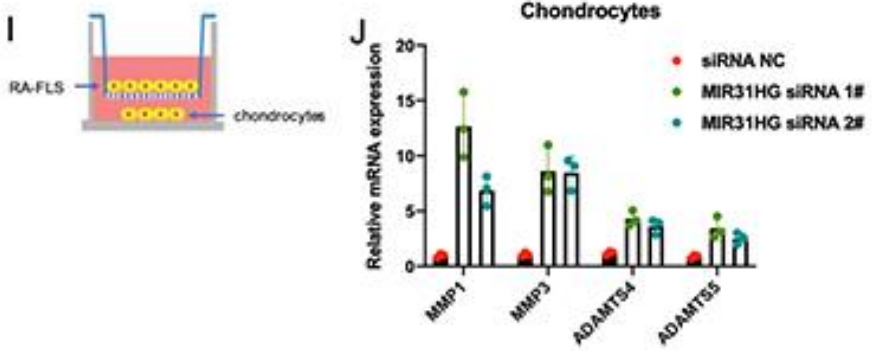

Figure 2. The effects of IncRNA MIR31HG on RA-FLS-mediated inflammation. (A) The effects of tocilizumab on endogenous expression of MIR31HG in RA-FLS ( $n=3$ ). (B-E) MIR31HG knockdown affects proliferation, metastasis, and production of inflammatory molecules and MMPs in RA-FLS $(n=3)$. (F-J) RA-FLS with reduced MIR31HG expression regulates primary macrophages and chondrocytes $(n=3)$. Data represent the mean $\pm S E M ; * p<0.05$, Student's $t$-test. 
A miR-214 expression

D
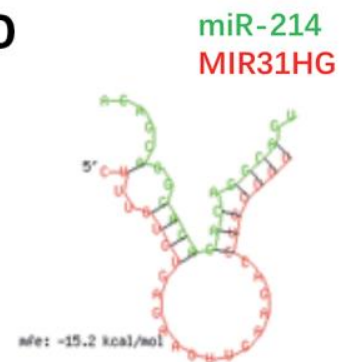

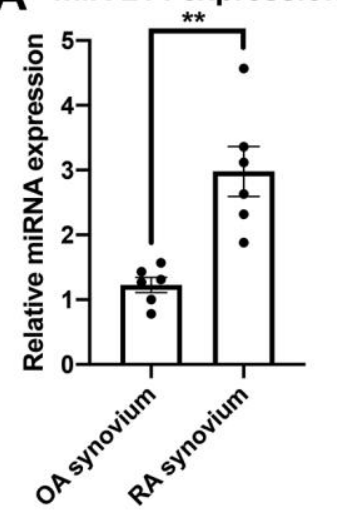

B miR-214 in RA-FLS

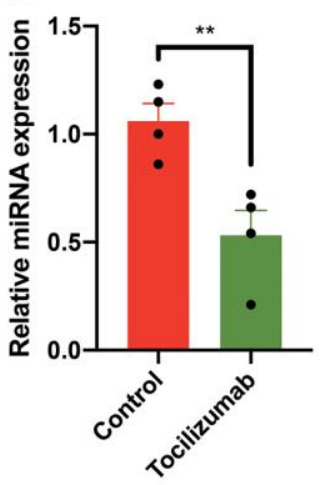

C miR-214 in RA-FLS

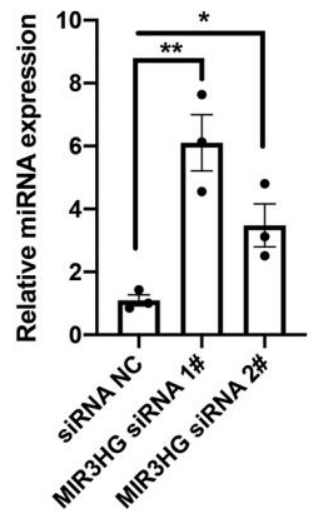

E MIR31HG in RA-FLS

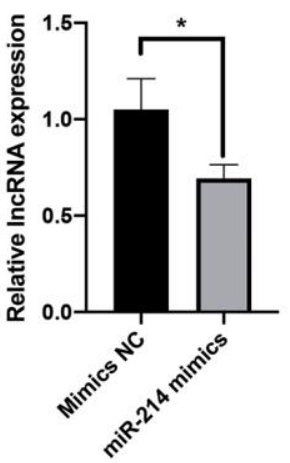

F Luciferase reporter assay
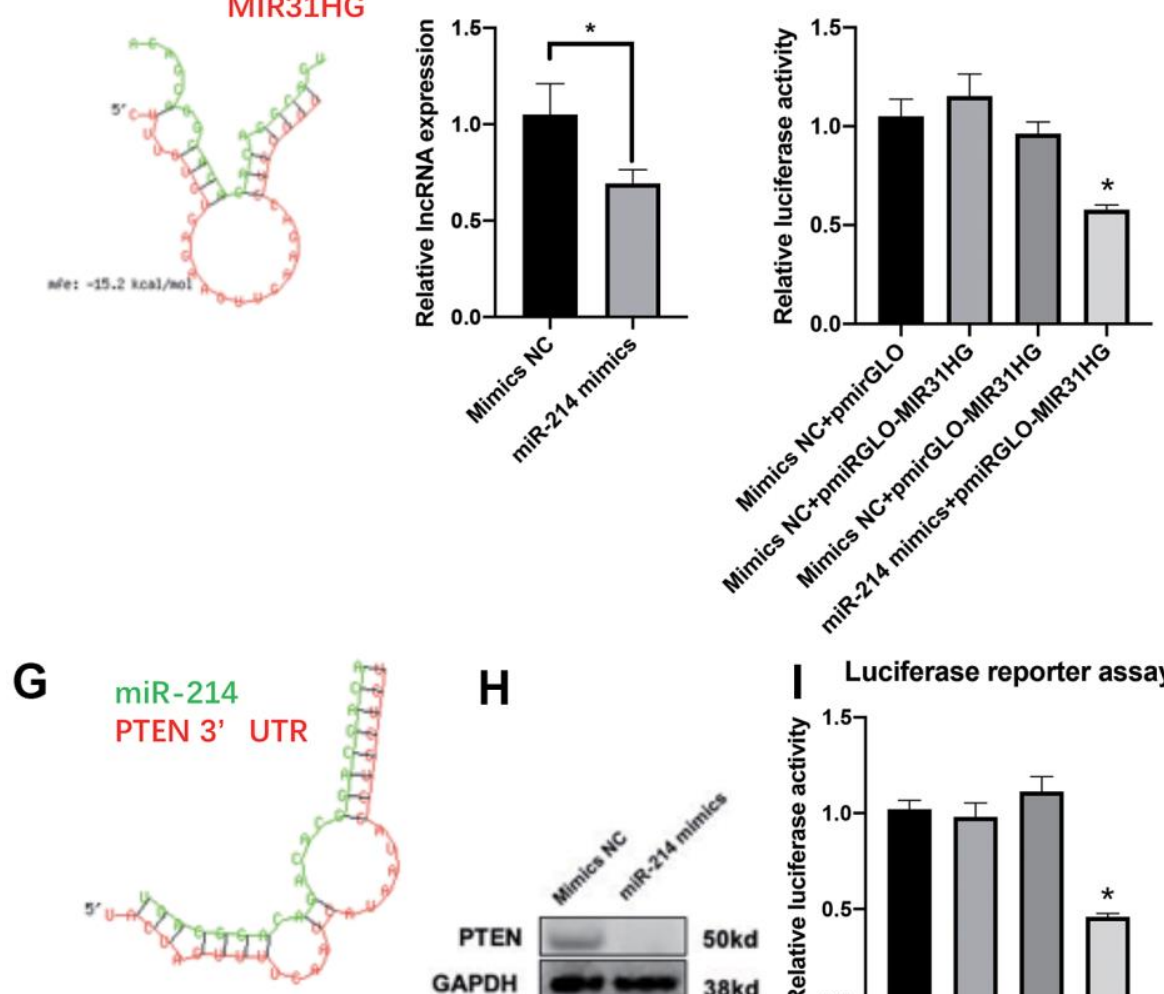

net $-18.9 \mathrm{kcal} / \mathrm{mol}$
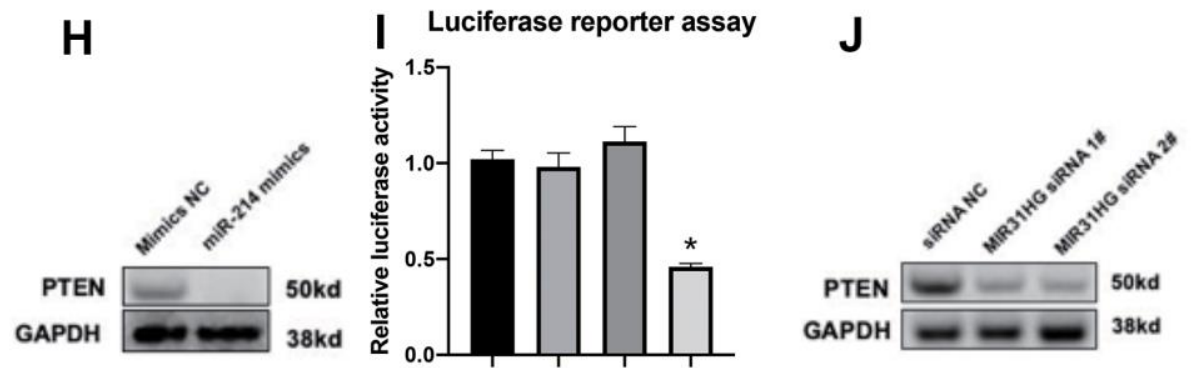

Figure 3. The ceRNA network for MIR31HG-miR-214-PTEN in RA-FLS. (A) The endogenous expression of miR-214 in OA and RA synovium samples $(n=6)$. (B) The endogenous expression of miR-214 in RA-FLS following tocilizumab treatment $(n=4)$. (C) The effects of MIR31HG siRNAs on miR-214 expression in RA-FLS ( $n=3$ ). (D) Bioinformatic prediction of binding between MIR31HG and miR-214. (E) The expression of MIR31HG in miR-214 overexpressing RA-FLS ( $n=3)$. ( $F)$ Luciferase reporter assay demonstrating the direct interactions between miR-214 and MIR31HG ( $n=3)$. (G) Bioinformatic prediction of binding between the PTEN 3' UTR and miR-214. (H) The expression of PTEN in miR-214 overexpressing RA-FLS $(n=3)$. (I) Luciferase reporter assay demonstrating the direct interactions between miR-214 and the PTEN $3^{\prime}$ UTR $(n=3)$. (J) Western blot showing the endogenous expression of PTEN in MIR31HG knockdown RA-FLS ( $n=3)$. Data represent the mean \pm $\mathrm{SEM} ; * \mathrm{p}<0.05$, as determined by the Student's $t$-test. 
showed that tocilizumab, MIR31HG, and miR-214 regulated p-AKT in RA-FLS (Figure 5A-5C). Next, we used an AKT inhibitor to confirm the function of the AKT pathway in RA-FLS. PI3K inhibitor LY294002 was used to block the AKT pathway in RA-FLS (Supplementary Figure 6) and it successfully inhibited the proliferation and migration of RA-FLS (Figure 5D, 5E). To validate that the AKT pathway is the downstream signaling pathway of MIR31HG, LY294002 was added to RA-FLS subjected to MIR31HG knockdown. The results of qPCR showed that the addition of LY294002 rescued MIR31HG knockdown-induced expression of inflammatory molecules in RA-FLS (Figure 5F) and restored the MIR31HG knockdown-induced inflammatory phenotype of primary macrophages and chondrocytes in the co-culture system (Figure 5G-5J). This suggests that the inhibitory effects of MIR31HG on RA-FLS mediated inflammation are at least partly mediated by suppressing the AKT pathway in these cells.

\section{DISCUSSION}

Several studies have suggested that tocilizumab exerts anti-inflammatory effects by inhibiting the expression of both inflammatory chemokines and adhesion molecules, such as MCP-1, IL-8, and ICAM-1 [14]. In this study, we investigated the anti-inflammatory effects of tocilizumab in RA-FLS from the perspective of ncRNAs, including lncRNAs and miRNAs. Our results revealed that tocilizumab inhibited RA-FLS inflammation by increasing the expression of the lncRNA MIR31HG. In addition, silencing MIR31HG inhibited the proliferation, migration, and expression of inflammatory factors and MMPs in RA-FLS by inhibiting the miR-214-PTEN-AKT pathway. In addition, co-culture experiments with chondrocytes and macrophages showed that MIR31HG indirectly mitigated the damage to chondrocytes and the inflammatory response in macrophages. Thus, our results suggest that MIR31HG acts as a regulator of the anti-inflammatory response in RA-FLS, thereby suppressing increased cell proliferation, invasiveness, and inflammation associated with RA. Taken together, our data suggest that MIR31HG plays a protective role in RA-induced synovitis (Supplementary Figure 7).

The invasiveness of the FLS is one of the major causes of bone and cartilage damage in RA. To date, it is not clear how tocilizumab and MIR31HG modulate
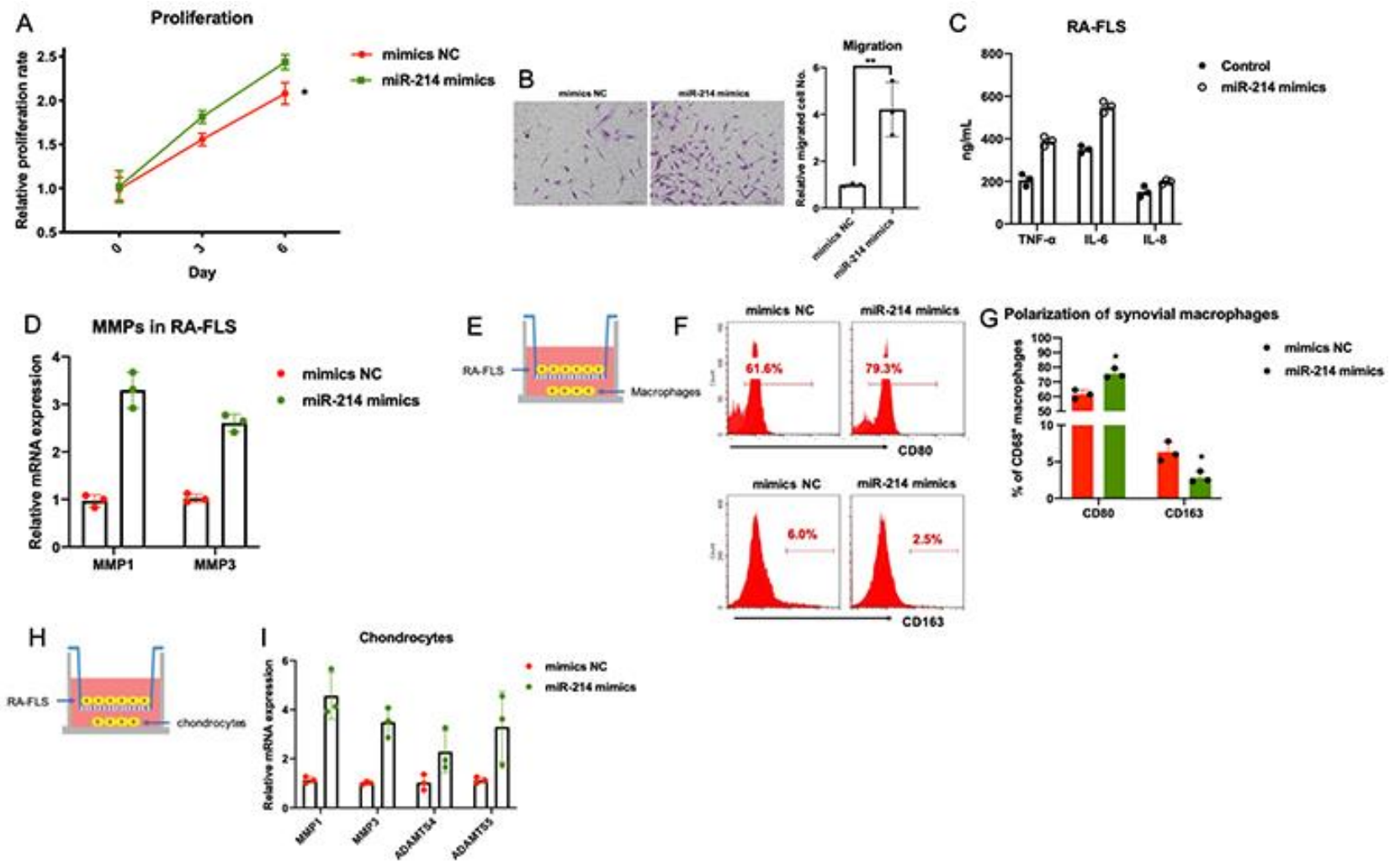

Figure 4. The effects of miR-214 overexpression on RA-FLS-mediated inflammation. (A-D) The effects of miR-214 overexpression on proliferation, metastasis, and production of inflammatory molecules and MMPs in RA-FLS ( $n=3$ ). (E-I) The effects of miR-214 overexpressing RA-FLS on primary macrophages and chondrocytes $(n=3)$. Data represent the mean \pm SEM; ${ }^{*} p<0.05$, Student's $t$-test. 
RA-FLS involved in joint degradation. Our findings shows that knocking down MIR31HG in primary RAFLS promotes chondrocyte degradation. Similarly, MMP overexpression is one of the main factors responsible for pathological ECM reconstruction and cell migration in the joints of patients with RA. MIR31HG knockdown enhanced the expression of MMPs, including MMP1 and MMP3, suggesting that MIR31HG, is a positive downstream regulator of tocilizumab and may mitigate pathological features of RA by inhibiting the invasiveness of RA-FLS.

Competing endogenous RNAs (ceRNAs) are one of the most important regulatory agents for modulating
lncRNA function. MIR31HG is a direct target of miR214 in non-small cell lung cancer (NSCLC) [10], and miR-214 promotes inflammation and immunity in several systems, such as organ transplant and immune tolerance $[15,16]$. In addition, PTEN is a direct target of miR-214 [17], and several studies have confirmed that PTEN plays an anti-inflammatory role in RA $[12,13$, 18]. These findings combined with our bioinformatic, qPCR, western blot, and dual luciferase reporter assays, suggest that a ceRNA regulatory network comprising MIR31HG-miR-214-PTEN is involved in RA-FLS. These findings expands our understanding of the molecular mechanisms underlying RA-FLS-mediated inflammation. The AKT signaling pathways plays
A

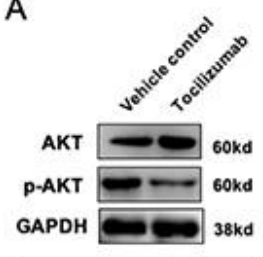

D Proliferation
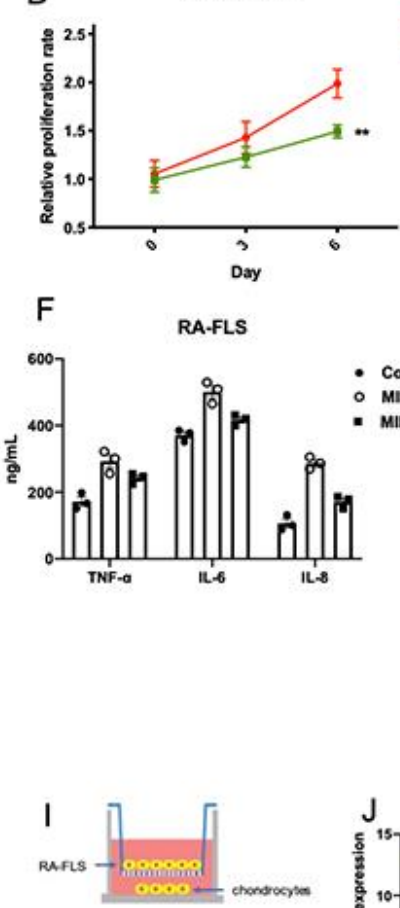

B

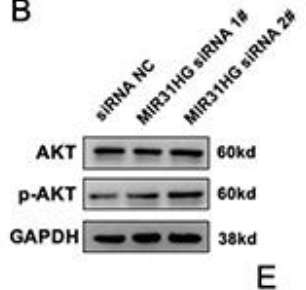

C

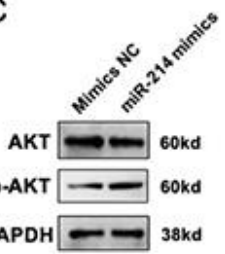

E

$\rightarrow$ OMSO

- LY294002

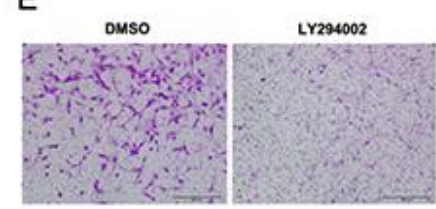

G

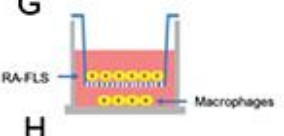

$\mathrm{H}$
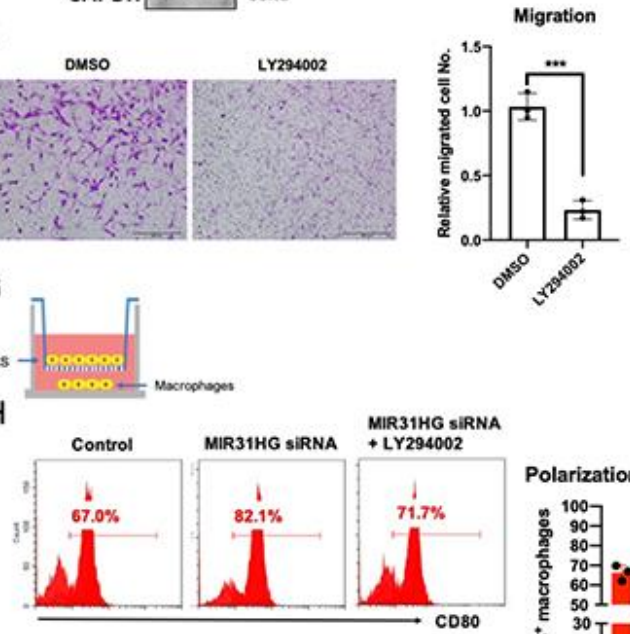

Polarization of synovial macrophages

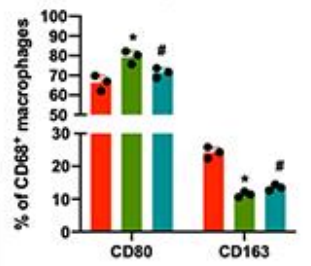

- Control

- mir31hg sirna

- MIR31HG siRNA + LY294002

Figure 5. The effects of the tocilizumab-MIR31HG axis on the AKT pathway in RA-FLS. (A-C) The effects of tocilizumab, MIR31HG and miR-214 on the AKT pathway in RA-FLS. (D, E) The effects of AKT pathway inhibitor, LY294002 on the proliferation and migration of primary RA-FLS. (F) LY294002 mediated rescue of the MIR31HG knockdown-induced expression of inflammatory molecules in RA-FLS. (G-J) LY294002 mediated rescue of the MIR31HG knockdown-induced expression of inflammatory phenotypes in primary macrophages and chondrocytes using an in vitro co-culture system. Data represent the mean $\pm \mathrm{SEM} ;{ }^{* *} \mathrm{p}<0.01$, as determined by the Student's $t$-test. 
pro-inflammatory role in RA-FLS [19], but little has been reported on its upstream regulation. Here, we report that tocilizumab and MIR31HG inhibit the activation of the AKT pathway in RA-FLS, and that this effect is mediated, at least in part, via the MIR31HGmiR-214-PTEN ceRNA regulatory network. These data provide new insights on the anti-inflammatory mechanism of tocilizumab and suggest potential targets for RA therapeutics.

In the future, we intend to expand this investigation in several ways. First, since tocilizumab is an IL-6R antagonist, we would like to study the relationship between IL-6 and MIR31HG, and identify the direct regulator of MIR31HG in the IL-6 signaling pathway. Our data showed that MIR31HG knockdown significantly increased IL-6 production (Figure 2D) and MIR31HG expression was induced in RA-FLS subjected to IL-6 knockdown (Supplementary Figure 8), suggesting the existence of a negative feedback loop between these two effectors. Furthermore, the number of RA patients included in this study was relatively small; therefore, we will continue to recruit more RA patients and healthy donors to validate our results in a larger clinical cohort. Finally, we plan to use murine arthritis models to further assess the effects of the tocilizumab-MIR31HG-miR-214-PTEN-AKT pathway on RA inflammation in vivo.

\section{MATERIALS AND METHODS}

\section{Patients}

The synovial tissue samples were obtained from patients with RA (all Chinese) who underwent synovectomy at the First Affiliated Hospital of Anhui Medical University. The current experiments were approved by the Ethics Committee of the First Affiliated Hospital of Anhui Medical University. The diagnosis of OA (six female patients, average age $=54.5$ years, age range: 48 63 years) and RA (one male and five female patients, average age $=56.67$ years, age range: 50 64 years) conformed to the criteria of the American College of Rheumatology (ACR) [20]. Trauma, sepsis, or tuberculosis-induced $\mathrm{OA}$ and RA were excluded from this study and none of the patients included in this study took any medications before their corrective surgery.

\section{Isolation and in vitro culture of FLS, macrophages and chondrocytes from patients with $R A$}

Macrophage, chondrocyte, and FLS isolation from RA synovial tissues was performed as previously described [21]. Briefly, the synovial membrane was excised from the OA and RA patients, and minced in Dulbecco's modified Eagle medium (DMEM; Gibco) containing
$10 \%$ fetal bovine serum (FBS; Gibco) and streptomycin/penicillin (Gibco). The synovial tissue was then digested in DMEM medium containing collagenase type IV (1 mg/mL; Sigma Aldrich) and 0.1 $\mathrm{mg} / \mathrm{mL}$ deoxyribonuclease I (Sigma Aldrich), and incubated at $37^{\circ} \mathrm{C}$ for $2 \mathrm{~h}$. The digested tissues were vortexed and resuspended in DMEM medium before centrifugation at 3,000 rpm for 5 min before being resuspended in PBS and subjected to cell sorting. Sorted For RA synovium chondrocytes, the cartilage from RA patients was minced and then treated with collagenase II (Sigma) with agitation $(200 \mathrm{rpm})$ at $37^{\circ} \mathrm{C}$ for $8 \mathrm{~h}$. The cell suspension was filtered by using a cell strainer $(40 \mu \mathrm{m})$, washed with PBS, centrifuged at 3,000 rpm for $10 \mathrm{~min}$, resuspended in $10 \mathrm{~mL}$ DMEM supplemented with 10\% FBS and penicillin/streptomycin, and then routinely cultured in a humidified incubator $\left(37^{\circ} \mathrm{C}\right.$ and $5 \% \mathrm{CO}_{2}$ ).

\section{Flow cytometry analysis of primary FLS and macrophages}

Isolated FLS were incubated with the antibodies specific to the following proteins at $4^{\circ} \mathrm{C}$ for $1 \mathrm{~h}$ to facilitate sorting: CD45-FITC(130-110-769, Miltenyi), CD31FITC(130-117-312, Miltenyi), CD146-FITC(130-097934, Miltenyi), CD235a-FITC(130-117-800, Miltenyi), hFAP $\alpha$-APC(FAB3715A-025, R\&D Systems), PDPNPE(130-117-799, Miltenyi), CD80-APC(130-117-719, Miltenyi), and CD163-APC(130-112-129, Milteny). Myeloid cells identified by CD45 expression, endothelial cells by CD31 expression, red blood cells by CD235a expression, and pericytes by CD146 were excluded. Two FLS markers (PDPN and FAR $\alpha$ ) were applied to identify FLS. For primary macrophage sorting, single cells from the RA synovium samples were centrifuged, the cell pellet was resuspended in fresh RPMI 1640 media, and sorted to obtain cells via CD45-FITC ${ }^{+} \mathrm{CD} 15-\mathrm{APC}^{-} \mathrm{CD} 1 \mathrm{c}-\mathrm{APC}^{-} \mathrm{CD} 14-\mathrm{PE}^{+}$strategy using FACSAria II cell sorter (BD Biosciences). Unlabeled cells were used as the negative control in the other flow cytometry experiments described in this paper. The sorted FLS were cultured in DMEM supplemented with $10 \%$ FBS and penicillin/streptomycin at $37^{\circ} \mathrm{C}$ and $5 \% \mathrm{CO}_{2}$ and the culture medium was replenished every two days, until cells reached $90 \%$ confluence. The other experiments from this study were completed using primary RA-FLS from passages 2 to 6 . Primary macrophages were cultured in Macrophage Base Medium XF (PromoCell) for 7-10 days prior to their use in any experiment.

\section{RA-FLS transfection}

RA-FLS were transfected with miR-214 mimics (100 $\mathrm{nM}$, sense sequence: 5'-ACAGCAGGCACAGACAGG 
CAGU-3', anti-sense sequence: 5'- UGCCUGUCUGU GCCUGCUGUUU-3') and the scrambled control (GenePharma) by using Lipofectamine RNAiMAX (Invitrogen). Cells were lysed 48-72 h after transfection and the lysates were used in the downstream experiments. Small interfering RNAs (siRNAs) against MIR31HG and IL-6 were purchased from GenePharma. The cell stimulation experiments were performed using RA-FLS treated with tocilizumab $(0.5 \mathrm{ng} / \mathrm{mL})$ and AKT pathway blocker, LY294002 $(20 \mu \mathrm{M} / \mathrm{mL})$.

\section{CCK-8 assay}

Cell proliferation was evaluated using the CCK-8 kit (DOJINDO) as per the manufacturer's protocol. Briefly, in a 96-well plate, cell suspensions were added to each well (5000 cells in $100 \mu \mathrm{L} /$ well) followed by the addition of $10 \mu \mathrm{L}$ CCK-8 reagent. Plates were incubated for $1 \mathrm{~h}$ before the absorbance was measured at $450 \mathrm{~nm}$ using a microplate reader.

\section{Cell migration assay}

Migration of FLS was evaluated using transwell assay (Corning), after they were treated with tocilizumab, MIR31HG siRNA, miR-214 mimics, and LY294002. The cells $(5,000$ cells/well) were seeded in the upper chamber of the transwell plate and incubated for $12 \mathrm{~h}$ in a humidified $\mathrm{CO}_{2}$ incubator at $37^{\circ} \mathrm{C}$. We evaluated the migration of the treated RA-FLSs by measuring their migration through the membrane of the upper chamber and the RA-FLSs attached to the lower membrane were stained with $0.1 \%$ crystal violet and visualized under a light microscope. The average number of migrated cells were estimated by counting the number of cells in six random fields of view.

\section{Real-time PCR}

RNA was extracted by using TRIzol (Invitrogen). The extracted RNA was washed and resuspended in nuclease-free water before being treated with DNase I. Total RNA was quantified and its purity was evaluated using a Nanodrop 2000 (Thermo Fisher Scientific). The mRNA reverse transcription kit (Takara) and TaqMan reverse transcription kit (Life Technologies) were used for reverse-transcribing mRNA and miRNA, respectively, as per manufacturer's instructions.

Universal SYBR Green Master mix (Applied Biosystems) was used to perform quantitative PCR (qPCR) for analyzing the mRNA expression, while Hairpin-itTM microRNA qPCR Quantitation Kit (GenePharma) was applied to detect miR-214 in RAFLS. All qPCR experiments were completed on a 7500 real-time PCR system (Applied Biosystems); GAPDH and U6 small nucleolar RNA (snoRNA) were used as endogenous controls for normalizing the expression of the mRNA and miRNAs, respectively.

\section{ELISA}

Production of inflammatory cytokines by the RA-FLS was evaluated using commercial ELISA kits. The supernatants were collected from each group and evaluated for TNF- $\alpha$, IL-6, and IL-8 expression using respective ELISA kits (R\&D Systems) according to the manufacturer's instructions.

\section{Western blot}

RA-FLSs were lysed using ice-cold lysis buffer, and total proteins were extracted and quantified using a protein quantification assay. Equal volumes of protein were mixed with loading buffer and resolved by performing SDS-PAGE. Resolved proteins were transferred to polyvinylidene fluoride membranes and then incubated with the following primary antibodies: anti-PTEN antibody (Abcam, 1:1000), anti-AKT antibody (Cell Signaling, 1:2000), and anti-p-AKT antibody (Proteintech, 1:2000). The membranes were then washed and incubated with horseradish peroxidaseconjugated secondary antibodies and the binding signal was visualized using an enhanced chemiluminescence system (GE Systems).

\section{RNA- and microRNA-seq}

Total RNA was extracted from the FLS following treatment with tocilizumab or transfection with MIR31HG siRNA or miR-241 mimics. The RNA quality and quantity was evaluated using a NanoDrop200 before being used to prepare the libraries for the RNAseq. For the miRNA-seq, total RNA was purified on a $15 \%$ urea denaturing gel, and small RNA regions, corresponding to the 18-30 nucleotide, were excised and recovered. These small RNAs were ligated to adenylated $3^{\prime}$ adapters annealed to unique molecular identifiers (UMI) followed by annealing to $5^{\prime}$ adapters. These adapter-ligated small RNAs were transcribed into cDNA using SuperScript II Reverse Transcriptase (Invitrogen), and then PCR amplified using specific primer cocktail and PCR mix to enrich the specific miRNA cDNA fragments. The PCR products were evaluated by agarose gel electrophoresis and those corresponding to 110-130 bp were purified using the QIAquick Gel Extraction Kit (QIAGEN). This library was (1) checked for the distribution of the fragment size using the Agilent 2100 bioanalyzer, and (2) quantified using qPCR (TaqMan Probe). The final PCR products were sequenced using the BGISEQ-500 platform (BGI). 


\section{Luciferase reporter assay}

We cloned $\sim 500$ bp of the MIR31HG and human PTEN 3'-untranslated region (3' UTR), which included the miR-214 seed sequence, into pmirGLO reporter vector (Promega) to produce luciferase reporter plasmids. HEK293T cells were plated in 12-well plates and transfected with $1 \mathrm{mg}$ of each reporter plasmid, $20 \mathrm{nM}$ of the miRNA mimics, or scrambled controls (NC). Luciferase activity was measured $48 \mathrm{~h}$ after transfection using the Dual-Luciferase Reporter Assay System (Promega) and GloMax 20/20 LUMINOMETER (Promega).

\section{Statistical analysis}

Normality of the data was checked and the statistical analysis was completed using SPSS software (version 13.0). As all samples were normally distributed, subsequent comparisons were completed using the Student's $t$-test, while the correlation analysis was performed using Pearson's analysis. Data was considered significant at $* \mathrm{p}<0.05, * * \mathrm{p}<0.01$ and $* * * \mathrm{p}<0.001$.

\section{Abbreviations}

RA: rheumatoid arthritis; FLS: fibroblast-like synoviocytes; ncRNA: non-coding RNAs; miR-214: micoRNA-214; MMPs: matrix metalloproteinases; ECM: extracellular matrix; ceRNA: Competing endogenous RNAs; NSCLC: non-small cell lung cancer; PAGE: polyacrylamide gel electrophoresis; qPCR: quantitative PCR; 3'UTR: 3'-untranslated region.

\section{AUTHOR CONTRIBUTIONS}

Conception and design: HMZ. Perform experiments, acquisition, analysis and interpretation of data: LC and HFJ. Writing, review, and/or revision of the manuscript: YJ, MJ, WGF and XYM. All authors read and approved the final manuscript.

\section{CONFLICTS OF INTEREST}

The authors declare that they have no conflicts of interest.

\section{FUNDING}

This work was supported by the Natural Science Foundation of Anhui Province, China, 2020 (KJ2020A0155).

\section{REFERENCES}

1. Guo Q, Wang Y, Xu D, Nossent J, Pavlos NJ, Xu J. Rheumatoid arthritis: pathological mechanisms and modern pharmacologic therapies. Bone Res. 2018; 6:15.

https://doi.org/10.1038/s41413-018-0016-9

PMID:29736302

2. Aletaha D, Smolen JS. Diagnosis and Management of Rheumatoid Arthritis: A Review. JAMA. 2018; 320:1360-72.

https://doi.org/10.1001/jama.2018.13103

PMID: $\underline{30285183}$

3. Tu J, Hong W, Zhang $P$, Wang $X$, Körner H, Wei W. Ontology and Function of Fibroblast-Like and Macrophage-Like Synoviocytes: How Do They Talk to Each Other and Can They Be Targeted for Rheumatoid Arthritis Therapy? Front Immunol. 2018; 9:1467. https://doi.org/10.3389/fimmu.2018.01467 PMID:29997624

4. Tu J, Wang X, Gong X, Hong W, Han D, Fang Y, Guo Y, Wei W. Synovial Macrophages in Rheumatoid Arthritis: The Past, Present, and Future. Mediators Inflamm. 2020; 2020:1583647. https://doi.org/10.1155/2020/1583647 PMID:32351318

5. Scheinecker C. The Role of T Cells in Rheumatoid Arthritis. In: Rheumatoid Arthritis. 2009. Chapter 8. pp. 91-6.

6. Volkov $M$, van Schie KA, van der Woude D. Autoantibodies and B Cells: The ABC of rheumatoid arthritis pathophysiology. Immunol Rev. 2020; 294:148-63.

https://doi.org/10.1111/imr.12829 PMID:31845355

7. Bustamante MF, Garcia-Carbonell R, Whisenant KD, Guma M. Fibroblast-like synoviocyte metabolism in the pathogenesis of rheumatoid arthritis. Arthritis Res Ther. 2017; 19:110. https://doi.org/10.1186/s13075-017-1303-3 PMID:28569176

8. Scott L. Tocilizumab: A Review in Rheumatoid Arthritis. Drugs. 2017; 77:1865-79. https://doi.org/10.1007/s40265-017-0829-7 PMID:29094311

9. Fang Y, Tu J, Han D, Guo Y, Hong W, Wei W. The effects of long non-coding ribonucleic acids on various cellular components in rheumatoid arthritis. Rheumatology (Oxford). 2020; 59:46-56.

https://doi.org/10.1093/rheumatology/kez472 PMID:31605483

10. Dandan W, Jianliang C, Haiyan H, Hang M, Xuedong L. Long noncoding RNA MIR31HG is activated by SP1 and promotes cell migration and invasion by sponging miR214 in NSCLC. Gene. 2019; 692:223-30. https://doi.org/10.1016/i.gene.2018.12.077 PMID:30659947 
11. García S, Liz M, Gómez-Reino JJ, Conde C. Akt activity protects rheumatoid synovial fibroblasts from Fasinduced apoptosis by inhibition of Bid cleavage. Arthritis Res Ther. 2010; 12:R33.

https://doi.org/10.1186/ar2941 PMID:20187936

12. Chen D, Liu D, Liu D, He M, Peng A, Xu J, Lin L, Luo F, Chen L, Huang X, Zhuang J, Xu J. Rheumatoid Arthritis Fibroblast-like Synoviocyte Suppression Mediated by PTEN Involves Survivin Gene Silencing. Sci Rep. 2017; 7:367.

https://doi.org/10.1038/s41598-017-00517-w

PMID:28337018

13. Li XF, Chen X, Bao J, Xu L, Zhang L, Huang C, Meng XM, $L i$ J. PTEN negatively regulates the expression of proinflammatory cytokines and chemokines of fibroblastlike synoviocytes in adjuvant-induced arthritis. Artif Cells Nanomed Biotechnol. 2019; 47:3687-96. https://doi.org/10.1080/21691401.2019.1661849 PMID:31842626

14. Suzuki M, Hashizume M, Yoshida H, Mihara M. Antiinflammatory mechanism of tocilizumab, a humanized anti-IL-6R antibody: effect on the expression of chemokine and adhesion molecule. Rheumatol Int. 2010; 30:309-15.

https://doi.org/10.1007/s00296-009-0953-0

PMID:19466425

15. Wang $Q$, Liu $Y$, Wu $Y$, Wen J, Man C. Immune function of miR-214 and its application prospects as molecular marker. PeerJ. 2021; 9:e10924.

https://doi.org/10.7717/peerj.10924 PMID:33628646

16. Gu C, Zhou XD, Yuan Y, Miao XH, Liu Y, Ru YW, Li KQ, Li G. MicroRNA-214 induces dendritic cell switching from tolerance to immunity by targeting $\beta$-Catenin signaling. Int J Clin Exp Pathol. 2015; 8:10050-60.

PMID:26617712
17. Bera A, Das F, Ghosh-Choudhury N, Mariappan MM, Kasinath BS, Ghosh Choudhury G. Reciprocal regulation of miR-214 and PTEN by high glucose regulates renal glomerular mesangial and proximal tubular epithelial cell hypertrophy and matrix expansion. Am J Physiol Cell Physiol. 2017; 313:C430-47.

https://doi.org/10.1152/ajpcell.00081.2017

PMID:28701356

18. Pap T, Franz JK, Hummel KM, Jeisy E, Gay R, Gay S. Activation of synovial fibroblasts in rheumatoid arthritis: lack of Expression of the tumour suppressor PTEN at sites of invasive growth and destruction. Arthritis Res. 2000; 2:59-64.

https://doi.org/10.1186/ar69

PMID:11219390

19. Malemud CJ. The PI3K/Akt/PTEN/mTOR pathway: a fruitful target for inducing cell death in rheumatoid arthritis? Future Med Chem. 2015; 7:1137-47.

https://doi.org/10.4155/fmc.15.55

PMID:26132523

20. Altman R, Asch E, Bloch D, Bole G, Borenstein D, Brandt K, Christy W, Cooke TD, Greenwald R, Hochberg M. Development of criteria for the classification and reporting of osteoarthritis. Classification of osteoarthritis of the knee. Diagnostic and Therapeutic Criteria Committee of the American Rheumatism Association. Arthritis Rheum. 1986; 29:1039-49. https://doi.org/10.1002/art.1780290816 PMID: $\underline{3741515}$

21. Tu J, Huang W, Zhang W, Mei J, Yu Z, Zhu C. TWIST1MicroRNA-10a-MAP3K7 Axis Ameliorates Synovitis of Osteoarthritis in Fibroblast-like Synoviocytes. Mol Ther Nucleic Acids. 2020; 22:1107-20. https://doi.org/10.1016/j.omtn.2020.10.020 PMID:33294296 


\section{SUPPLEMENTARY MATERIALS}

\section{Supplementary Figures}

A IncRNA candidates

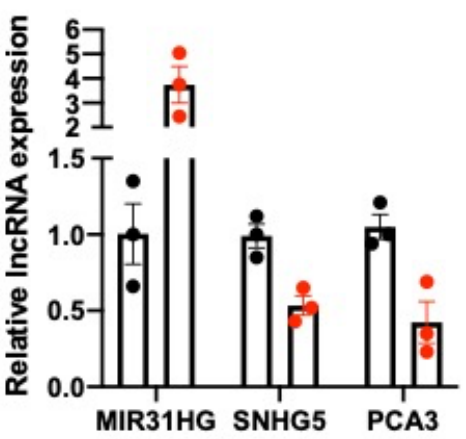

B miRnA candidates

- Control

- Tocilizumab

Supplementary Figure 1. The candidates of (A) IncRNAs (MIR31HG, SNHG5, PCA3) and (B) miRNAs (miR-214, miR-361, miR-575) were selected for experimental validation.
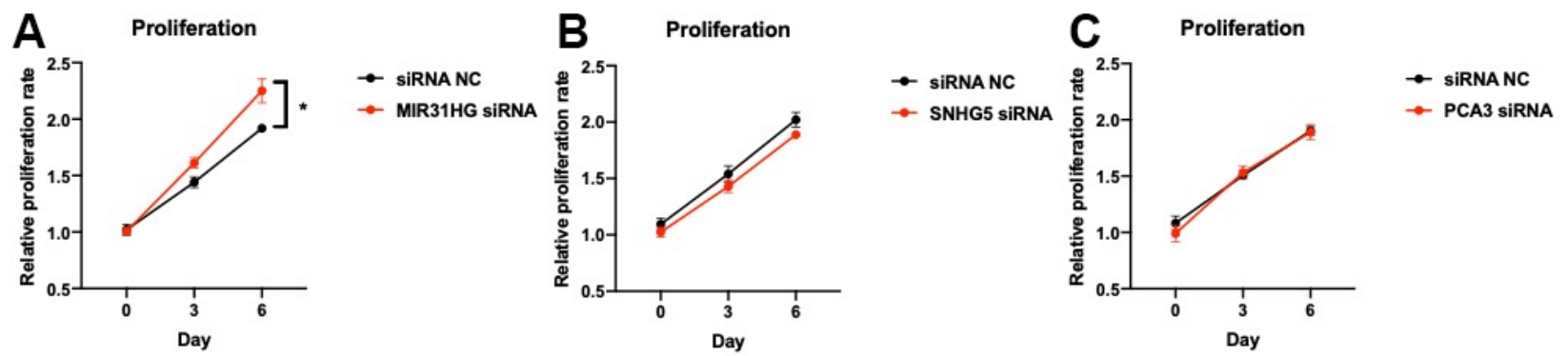

Supplementary Figure 2. The effects of knockdown of IncRNAs candidates (A) MIR31HG, (B) SNHG5 and (C) PCA3 on proliferation of RAFLS. Data represent means \pm SEM; ${ }^{*} p<0.05$, as determined by the Student's $t$ test.

RA-FLS

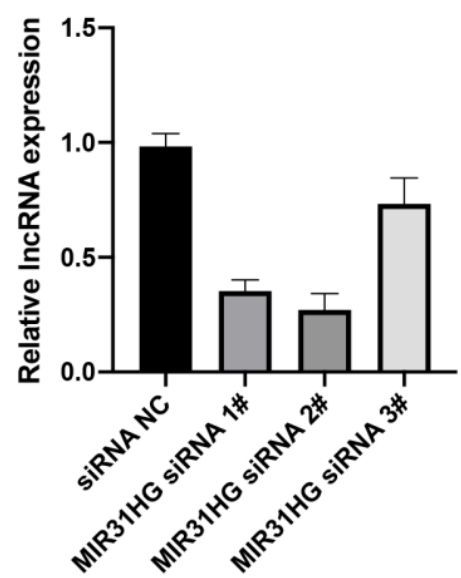

Supplementary Figure 3. The knockdown efficiency of MIR31HG siRNAs in RA-FLS. 


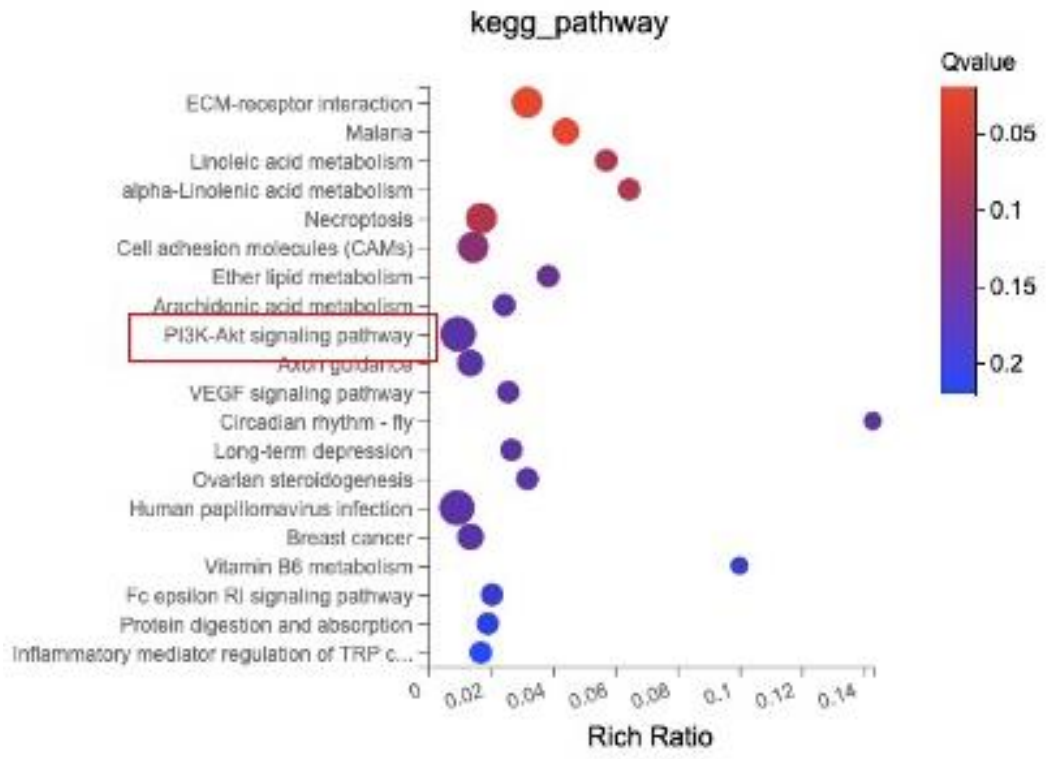

Supplementary Figure 4. The KEGG pathway analysis of miR-214 overexpression RA-FLS.

\section{Correlation analysis of MIR31HG and miR-214 in RA-FLS}

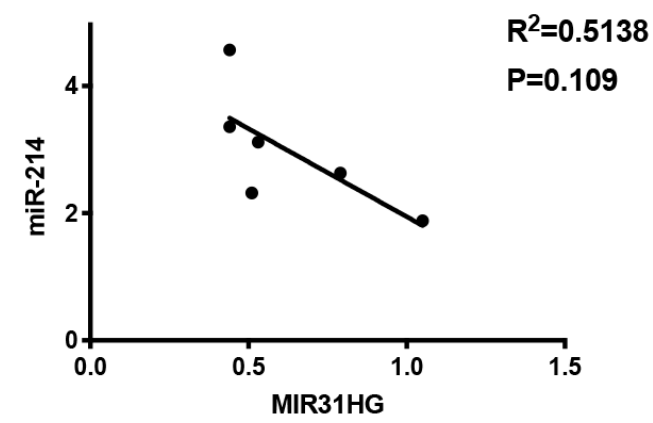

Correlation analysis of PTEN and miR-214 in RA-FLS

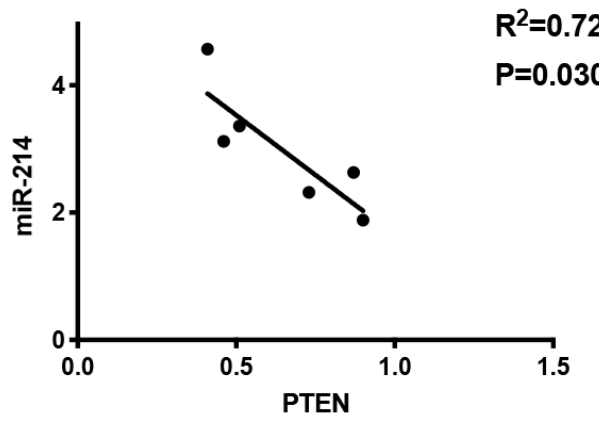

Supplementary Figure 5. The correlation coefficient between MIR31HG and miR-214 or PTEN in RA-FLS.

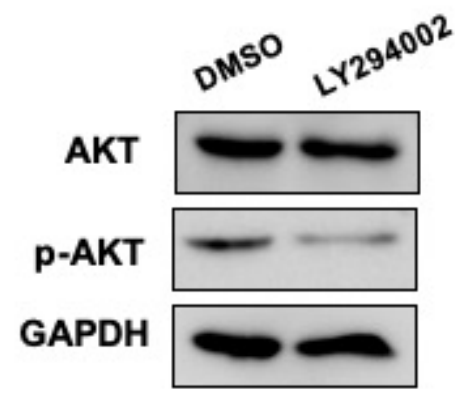

Supplementary Figure 6. The validation of the inhibitory effect of LY294002 on AKT pathway in RA-FLS. 


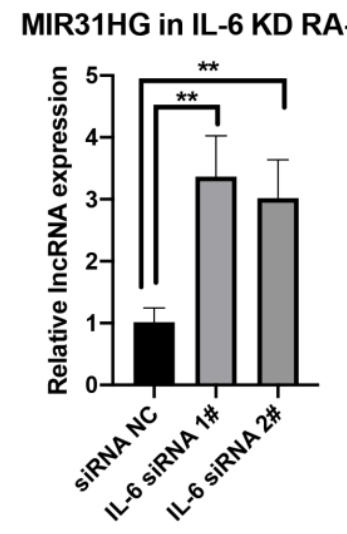

Supplementary Figure 7. The expression of MIR31HG in IL-6 KD RA-FLS.

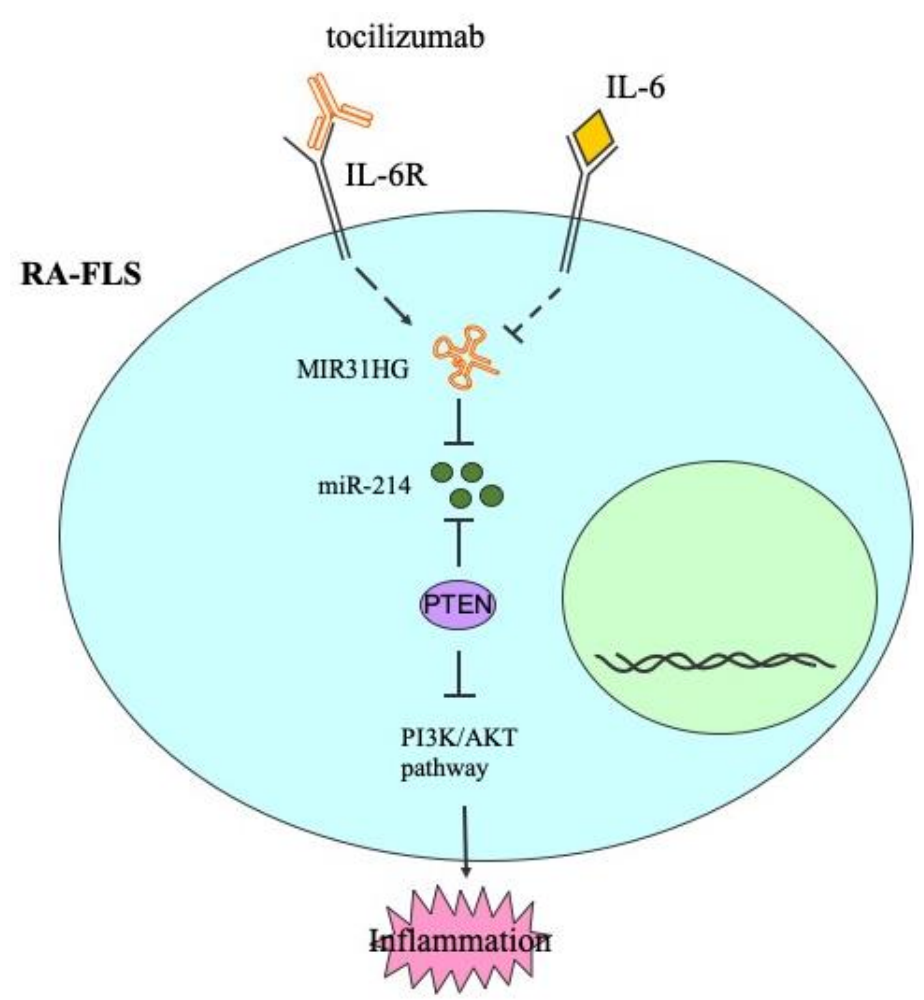

Supplementary Figure 8. The graphic abstract of the current manuscript. 


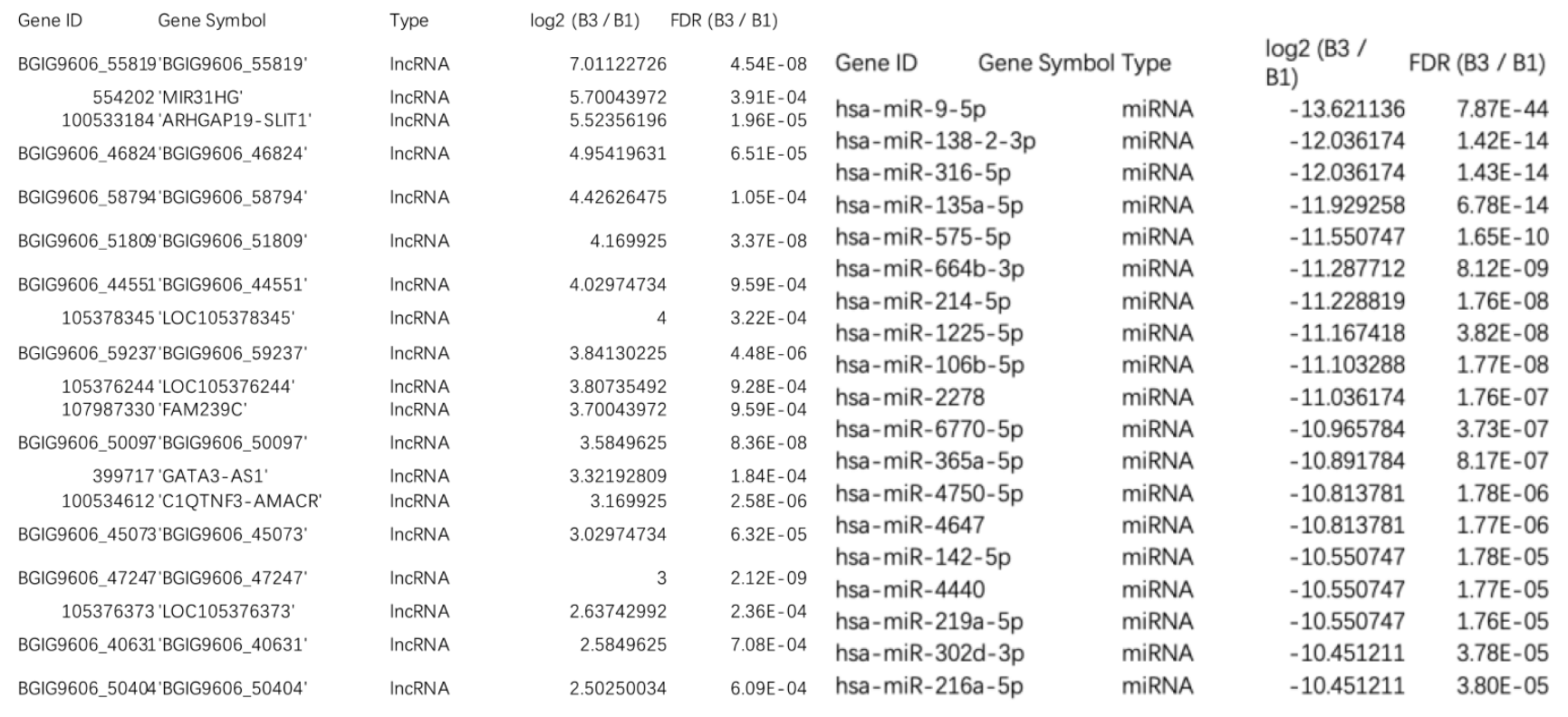

Supplementary Figure 9. Top 20 up-regulated IncRNAs and down-regulated miRNAs in tocilizumab-treated RA-FLS. 